EPJ Web of Conferences 87,04010 (2015)

DOI: $10.1051 /$ epjconf/ 20158704010

(c) Owned by the authors, published by EDP Sciences, 2015

\title{
Development of Resonant Diplexers for high-power ECRH - Status, Applications, Plans
}

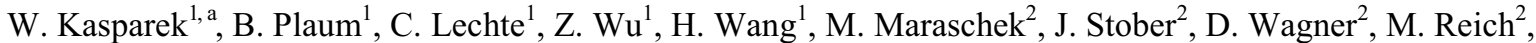 \\ M. Schubert ${ }^{2}$, G. Grünwald ${ }^{2}$, F. Monaco ${ }^{2}$, S. Müller ${ }^{2}$, H. Schütz ${ }^{2}$, V. Erckmann ${ }^{2}$, N. Doelman ${ }^{3}$, R. van den Braber ${ }^{3}$, \\ W. Klop ${ }^{3}$, H. van den Brand ${ }^{4}$, W. Bongers ${ }^{4}$, B. Krijger ${ }^{4}$, M. Petelin ${ }^{5}$, E. Koposova ${ }^{5}$, L. Lubyako ${ }^{5}$, A. Bruschi ${ }^{6}$, \\ K. Sakamoto ${ }^{7}$, teams at the contributing institutes, and ASDEX Upgrade Team \\ ${ }^{1}$ Institut f. Grenzflächenverfahrenstechnik und Plasmatechnologie, D-70569 Stuttgart, Germany \\ ${ }^{2}$ Max-Planck-Institut für Plasmaphysik, EURATOM Ass., 85748 Garching and 17491 Greifswald, Germany \\ ${ }^{3}$ Department of OptoMechatronics, TNO Tech. Sciences, NL-2600 AD, Delft, The Netherlands \\ ${ }^{4}$ Dutch Institute For Fundamental Energy Research, NL-3439 Nieuwegein, The Netherlands \\ ${ }^{5}$ Inst. of Applied Physics, Russian Academy of Science, 603950 Nizhny Novgorod, Russia \\ ${ }^{6}$ Istituto di Fisica del Plasma, EURATOM-ENEA-CNR Ass., I-20125 Milano, Italy \\ ${ }^{7}$ Japan Atomic Energy Agency (JAEA), 801-1, Mukoyama, Naka, Ibaraki 311-0193, Japan
}

\begin{abstract}
The development of diplexers for ECRH has been pursued at a number of institutes because of their attractive variety of applications: Power combination, non-mechanical, electrically controlled switching (of combined beams) between launchers with tens of $\mathrm{kHz}$, and discrimination of low-power ECE signals from high-power ECRH is feasible. In a first part, this paper reports on plasma experiments with a ring resonator (Mk IIa) at ASDEX Upgrade. Commissioning experiments on fast switching between two launchers for synchronous stabilization of neoclassical tearing modes, as well as in-line ECE measurements have been performed, and experimental issues and first results are discussed. A clear influence of the switching phase on the amplitude of the $3 / 2$ NTM mode was measured, complete stabilization could, however, not be demonstrated yet mainly due to imperfect resonator control. Concepts for improved tracking of the diplexers to the gyrotron frequency are presented. In a second part, the design of diplexers with ring resonators matched to HE11 fields is briefly discussed; these devices can be connected to corrugated waveguides without any mode converters. A compact version (MQ IV) is under investigation, which is compatible with the ITER ECRH system $(170 \mathrm{GHz}, 63.5 \mathrm{~mm}$ waveguide, vacuum tight casing), with the final goal of high-power tests at the 170 GHz gyrotron facility at JAEA in Naka, Japan. First low-power test results are presented.
\end{abstract}

\section{Introduction}

In the past years, high-power diplexers have become of growing interest for applications in ECRH systems. Different variants [1] have been investigated, and especially ring resonator (Fig. 1, left) diplexers [2] have demonstrated high versatility. The example of the 170 GHz/24 MW ITER ECRH system [3], where the power can be switched between the equatorial launcher (EL) and the upper launchers (UL) by waveguide switches, illustrates the many options. If the mechanical switches were replaced by four-port resonant diplexers, the power could be (i) arbitrarily distributed between the launchers according to the physics needs by simply tuning the resonance frequency, while the gyrotrons run continuously. For synchronous NTM stabilization [4], a gyrotron voltage modulation synchronous to the rotation frequency of the islands together with a proper tuning of the diplexer would (ii) strongly amplify the amplitude modulation in the UL and thus yield high stabilization efficiency. In this context, the mode purification characteristics of the resonator could (iii) improve the focussing and reduce the possible beam squint of the UL. The filtering of very-high-order modes would (iv) reduce the thermal loading of the sensitive cuffs of the vacuum windows in the UL as well as in the EL. A stray radiation detector in the diplexer would (v) serve as a monitor for mode purity. The isolated input port could be used to (vi) receive ECE radiation from the plasma by sharing the UL with the (forward) ECRH [5]. Eventually, if an upgrade of the ECRH power is needed, these second input ports could be (vii) used to connect further gyrotrons, as the diplexer can operate as power combiner [6].

All these features are based on the transmission characteristics (Fig. 1, right) [6], namely narrow

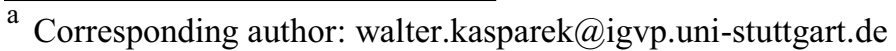


resonances in output 2 ("resonant channel"), which are periodic with $\mathrm{c} / L$, and broad transmission regions in between ("non-resonant channel"). The width of the resonances is determined by the efficiency $R_{1}$ of the coupling gratings (Fig. 1, left). With a control of $L$ by making one of the resonator mirrors movable (linear motion needed is less than one wavelength) $[6,7]$, the diplexer can be tuned with respect to the gyrotron frequency according to the application, especially to point A, where the gyrotron frequency $f_{\text {gyr }}$ is resonant (tracking to dip), and point $\mathrm{B}$, where $f_{\mathrm{gyr}}$ corresponds to the slope of the resonance (tracking to slope).
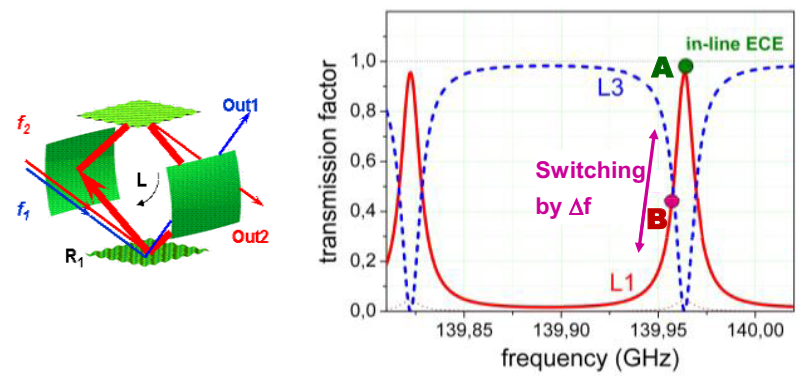

Figure 1. Left: Principle of a ring resonator diplexer. Right: Transmission functions for the resonant (OUT2, red, solid) and the non-resonant (OUT1, blue, dashed) output, calculated for the parameters of diplexer Mk IIa with a resonator length of $L=2.212 \mathrm{~m}$, and a grating coupling efficiency of $R_{1}=0.22$. The pink dot shows the operation point $\mathrm{B}$ for fast switching generated by a small frequency modulation.

In this paper, attempts to stabilize NTM modes on ASDEX Upgrade by fast switching between two launchers are described in chapter 2. An improved concept for resonator control is discussed in chapter 3 . Meanwhile, more compact diplexers have been built; in chapter 4, first tests are presented for the version MQ IV, which is compatible with the ITER ECRH.

\section{Experiments to stabilize NTM modes at ASDEX Upgrade}

\subsection{Experimental set up}

In the tokamak ASDEX Upgrade, H-mode discharges with high-power NBI often feature neoclassical tearing modes. They can be stabilized by ECCD driven in the Opoint of the magnetic islands [4], which at ASDEX Upgrade rotate with a frequency between 10 and $30 \mathrm{kHz}$. For proper focussing and deposition radius, $700 \mathrm{~kW} \mathrm{CW}$ power or $400 \mathrm{~kW}$ average power with power modulation synchronously to the rotation of the islands are needed to stabilize 3/2 NTM modes [8].

To demonstrate the applicability of diplexers for NTM stabilization via synchronous switching between two launchers, the diplexer Mk II was integrated into the corrugated waveguide transmission of the ECRH-2 system at ASDEX upgrade [9, 10]. One input was connected to gyrotron Gy3; the outputs were feeding the launcher L3 via the non-resonant channel, and launcher L1 via the resonant channel. To match the polarisation to the launching geometry, mitre-bend polarisers were installed behind the diplexer. The signals from the directional couplers installed in the transmission lines near to the launchers were used as input for the control of the resonator mirror drive; the controller was set for frequency tracking to operation point $\mathrm{B}$ on the positive (low-frequency) slope of the resonance. The set-up is shown in Fig. 2.

With this set-up, various commissioning experiments had been performed to test the performance of the system. Especially, effective switching between two launchers with different deposition radii could be demonstrated by analysis of the heat waves generated in the plasma [11].

The basic setting of the launchers for the NTM stabilization experiments is shown in Fig. 3.

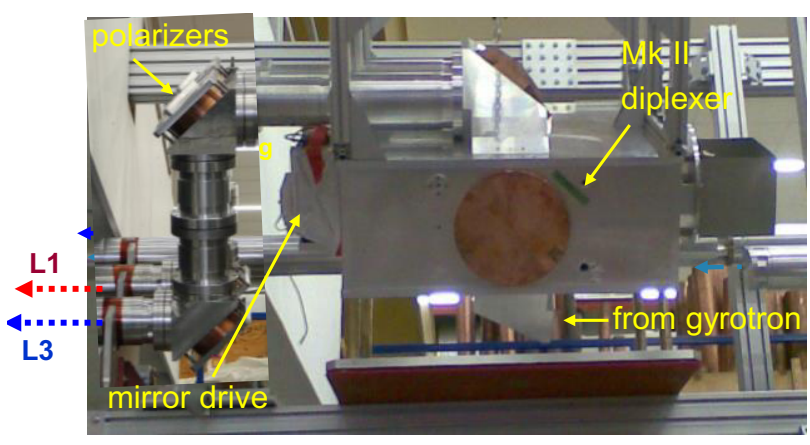

Figure 2. Installation of the diplexer MkII in the ECRH2 system at ASDEX Upgrade. The two output waveguides are connected via mitre bend polarizes to the launchers L1 and L3. The mirror drive connected to one of the resonator mirrors is used to track the diplexer frequency characteristics to the gyrotron frequency.

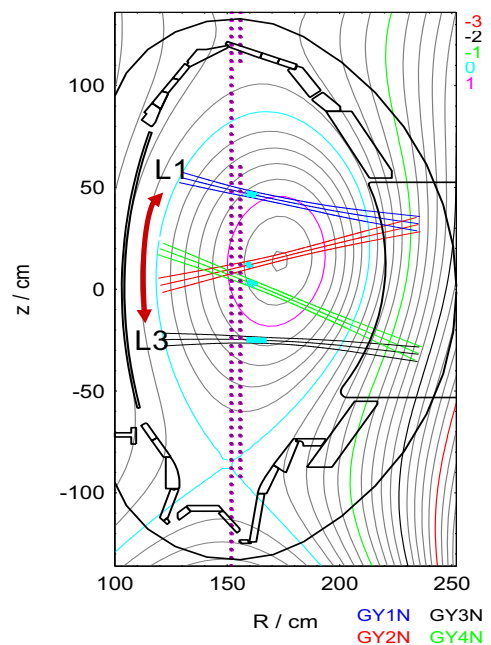

Figure 3. Launching geometry for the ECRH2 on ASDEX Upgrade. Two beams from launchers L1 and L3 are used for stabilisation of NTMs, two other gyrotrons are used for central heating. The dotted line marks the $\mathrm{EC}$ resonance, blue regions show the EC power deposition region. 


\subsection{Experimental results}

For the experiments, H-mode discharges with strong NBI heating (12.5 MW), and central ECRH (1.8 MW) were used, as seen in Fig. 4. When a 3/2 NTM mode was destabilized, the NBI power was reduced to $7 \mathrm{MW}$. From $\mathrm{t}=2.3 \mathrm{~s}$ to $\mathrm{t}=6.3 \mathrm{~s}$, gyrotron Gy 3 was switched on with a typical average power of $500 \mathrm{~kW}$.

The toroidal angle of injection of the launchers L1 and L3 were set to $9^{\circ}$, and the poloidal injection angles were programmed such that the power deposition radii for the two launchers scanned across the $q=1.5$ surface in two poloidally symmetric positions, such that the O-point and the X-point of the m:n=3:2 island was alternatively in front of L1 and L3 (see Fig. 3).

The gyrotron was voltage modulated with an amplitude of $3.5 \mathrm{kV}$, corresponding to a gyrotron frequency variation of about $14 \mathrm{MHz}$, enough to switch the power between L1 and L3 at a frequency derived from a signal of the Mirnov coils system, synchronous to the $3 / 2$ NTM modes. Here, the phase shifter between Mirnov signal and the gyrotron modulator was set such that the switching of the power between the launchers produced an ECCD which was maximum alternatively in L1 and L3, but always in the O-point of the rotating islands.

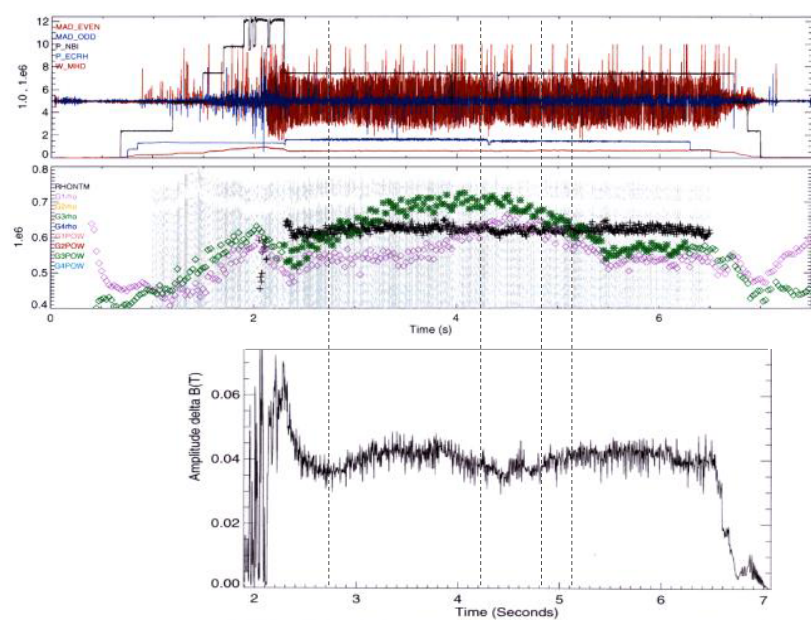

Figure 4. ASDEX Upgrade shot $\# 29570$, showing the heating power and the 3/2 NTM (top). Black: NBI; blue: ECRH; brown: NTM signal. In the middle, the location of the power deposition wrt. $\mathrm{r}_{\mathrm{NTM}}$ is shown. Magenta: deposition radius of launcher L1; green: deposition radius for L3; black: $\mathrm{r}_{\mathrm{NTM}}$. At the bottom, the NTM amplitude is shown, with dashed lines giving the times when the power deposition coincides with the radius of the NTMs.

Fig. 4 (bottom) shows the effects on the mode amplitude. One can see a reduction of the NTM, when the power deposition coincides with the radius where the NTMs are located. However, the stabilization effect is relatively weak. In total, three successful discharges were done with identical parameters, two with optimum phasing for ECCD in the O-points, and one shot with anti-phasing to get maximum current in the X-points of the islands, i.e. possibly a destabilizing effect. The comparison of the shots (Fig. 5) shows a clear difference of the behaviour of the mode amplitude, and especially in the first half of the discharge confirms the effects expected for ECCD in the O-point or the X-point.

Reasons for the fact that the stabilization is significantly less compared to the reference shot (synchronous $100 \%$ power modulation with $410 \mathrm{~kW}$ average power, launched from a single antenna) are manifold: (i) The switching contrast was only $60 \%$ to $80 \%$ for the given gyrotron frequency modulation. (ii) The time for the perfect overlap of power deposition location and $\mathrm{r}_{\mathrm{NTM}}$ might have been too short. (iii) During the shot, usually only one antenna launched to $r_{\text {NTM }}$ at a given time, therefore less than $250 \mathrm{~kW}$ were available for stabilization. Note that a true demonstration experiment of the diplexer capabilities to increase the efficiency in the NTM mode stabilisation would require real-time mode position tracking with both the launchers, since otherwise stabilisation of one launcher may be masked by destabilisation of the other one. (iv) The mirror control suffered from e.m. interference, which probably was the main reason, and consequences are discussed in the next chapter.

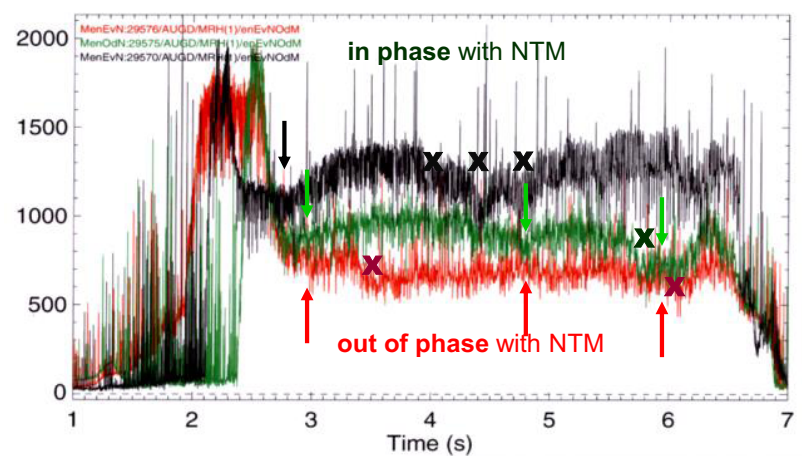

Figure 5. NTM amplitude for shots \# 29570 and 29575, where the phase of the switching was set for maximum stabilization of the NTM, (black, green), and shot \#29576 with anti-phasing for destabilization of NTM. The crosses mark short drop-outs of the NBI.

\section{Resonator control}

\subsection{Problems encountered during AUG experiments}

Analysis of the signals for power at the diplexer outputs and the mirror control showed a drift of the operation point during the pulses. Basically, the mirror drive uses the ratio (averaged over the modulation period) between the two outputs to L1 and L3 as control signal, to keep the operation point at the slope of the resonance ( $c f$. Fig.1). However, as the frequency modulation swing is comparable with the width of the resonance, a stable operation point with the frequency shift keying from the positive to the negative slope of the resonance can occur. In the experiments, this happened obviously, as can be seen in Fig. 6 from a zoom into the power monitor signals at the input and the outputs of the diplexer, respectively. At the first phase of the pulse $(\mathrm{t}<3 \mathrm{~s})$, the switching works correctly. At later phases of the pulse $(t>3 \mathrm{~s})$, the variation of the power is small, and - within the limits imposed by the too low sampling rate of the signals - a 
doubling in the switching frequency can be seen; this is a sign that the switching occurs between an operation point on the positive to a point at the negative slope of the diplexer resonance. Thus the effects of synchronous switching between launchers diminishes, as a result we have similar powers at both launchers.

The reason(s) which caused this drift of the operation point are not clear; one possibility which might have caused the problem is an abrupt change of the gyrotron frequency by a few $\mathrm{MHz}$.

$\mathrm{t}<3 \mathrm{~s}:$

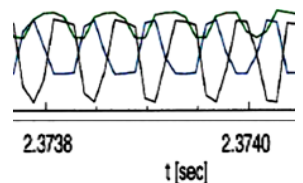

$\mathrm{t}[\mathrm{sec}]$ $\mathbf{t}>\mathbf{3}$ s:

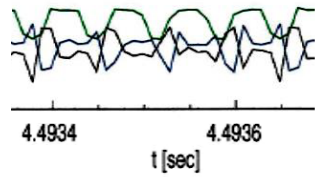

Figure 6. Power monitor signals for input power $\mathrm{P}(\mathrm{IN})$ and output powers to the launchers $\mathrm{P}(\mathrm{L} 1)$ and $\mathrm{P}(\mathrm{L} 3)$. Note that the modulation of $\mathrm{P}(\mathrm{IN})$ is due to the voltage modulation. At the beginning of the pulse (example $\mathrm{t}=2.37 \mathrm{~s}$, left), a clear switching from L1 to L3 with contrast or $60 \%$ (L1) and $80 \%$ (L3) is seen. At the later phase (example $t=4.49 \mathrm{~s}$, right), the switching occurs rather from the positive to the negative slope.

\subsection{Error signal generation for improved resonator frequency tracking}

To make the mirror control more robust, a new control scheme is developed (Fig. 7). It consists of a simple interferometer circuit using the outputs of the power monitors, which after appropriate balancing of phase and power are fed into the collinear ports of a magic $\mathrm{T}$. The difference of the powers at the H-plane and E-plane ports results is an error signal, which gives a zero-crossing at the resonance frequency (see Fig. 8).

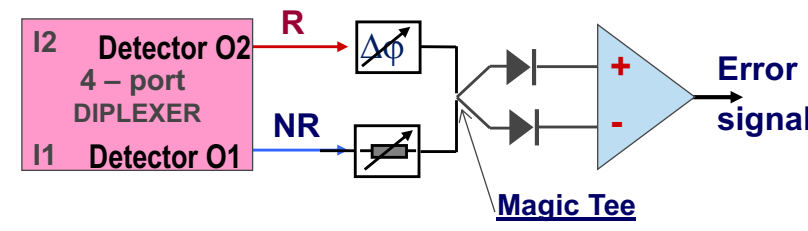

Figure 7. Generation of an error signal which provides a zerocrossing at resonance.

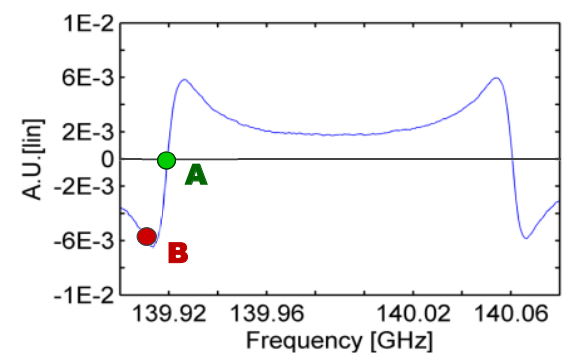

Figure 8. Error signal for the circuit in Fig. 7 as function of the frequency. Note that the points with zero-crossing correspond to the diplexer resonances.
This feature can be directly used to track the resonator to the dip (operation point A). The change of sign of the error signal gives information on the positive or negative slope, respectively, and thus will remove uncertainties in the tracking to slope.

For the realization of this control, power monitors close to each other with short waveguide connections are needed. With the present set up, this is not possible. However, more compact diplexers will have integrated couplers, where the integration of this controller is no problem.

\section{Compact diplexers with $\mathrm{HE}_{11}$ resonators}

\subsection{General issues}

Internally, the diplexer $\mathrm{Mk}$ IIa is operated with a Gaussian beam resonator, which requires transitions from $\mathrm{HE}_{11}$ to $\mathrm{TEM}_{00}$ at the inputs and outputs to match the device to the corrugated transmission lines. To minimize the number of components and thus to ease integration to transmission lines, compact diplexers have been developed. They use direct corrugated waveguide inputs, as matching optics and resonator mirrors for $\mathrm{HE}_{11}$ fields are used. The performance of the compact $\mathrm{MC}$ IIIb ( $L=0.906 \mathrm{~m}$ ) for $140 \mathrm{GHz}$, which is compatible with the ASDEX Upgrade system, has been presented in [11]. Meanwhile, a diplexer mock-up for $170 \mathrm{GHz}$ has been built, which is compatible with ITER ECRH.

\subsection{Design of MQ IV}

The design of MQ IV (for corrugated waveguide diameter of $63.5 \mathrm{~mm}$, and $170 \mathrm{GHz}$ ) is shown in Fig. 9. It consists of a massive Aluminium box with precise outer machining to accommodate the mirrors (two pairs of matching mirrors, two coupling gratings, and the lower resonator mirror), which simultaneously are used as vacuum flanges. The top plate of the box (not shown) carries the upper resonator mirror, which eventually will be equipped with a controlled mirror drive. The present mock-up features solid $\mathrm{Al}$ and $\mathrm{Cu}$ mirrors without cooling; however, cooling could be added later easily without extra vacuum feed-throughs. Both matching and the resonator mirrors are designed as phase-reversing elements for the fields radiated from and to the $\mathrm{HE}_{11}$ input/output waveguides, which are attached rigidly to the casing. The matching mirrors (double-sided roof-top design) image the waveguide aperture field at the input(s) onto the coupling gratings; a second matching mirror performs the imaging onto the apertures of the output waveguides. The (astigmatic) $\mathrm{HE}_{11}$ field on the grating is imaged via the resonator mirror onto the opposite grating. Shallow holographic gratings on the surface of the matching mirrors together with receiver horns mounted in the box serve as directional couplers. 



Figure 9. Design of the compact resonant diplexer MQ IV, showing the optical set up (top) with pictures of the vacuum box with one Aluminium matching mirror, the grating splitters, and one resonator mirror installed.

Dimensions as well as characteristic data are given in Table 1.

Table 1. Dimensions and basic characteristics of MQ IV.

\begin{tabular}{|l|c|}
\hline Frequency: & $170 \mathrm{GHz} \pm 0.5 \mathrm{GHz}$ \\
\hline Waveguide: & $63.5 \mathrm{~mm}$, ITER flanges \\
\hline Casing L x W x H: & $514 \times 580 \times 460 \mathrm{~mm}$ \\
\hline Input/output w.g. distance: & $300 \mathrm{~mm}$ \\
\hline Cavity length: & $1.075 \mathrm{~m}$ \\
\hline Distance of resonances & $279 \mathrm{MHz}$ \\
\hline $\begin{array}{l}\text { Wrong mode suppression of } \\
\text { resonant output, } \\
\text { low-order modes: } \\
\text { very-high-order modes: }\end{array}$ & $8 \mathrm{~dB}$ (average value) \\
\hline $\mathrm{HE}_{11}$ isolation (multi-mode): & $>40 \mathrm{~dB}$ \\
\hline
\end{tabular}

\subsection{Low-power test}

At present, the diplexer MQ IV is being characterized using a network analyzer (ABmm 8-350), and lens horns as mode generators.



Figure 10. Transmission functions for MQ IV. Solid lines: measured transmission for the resonant (red) and the nonresonant (blue) channel. Dashed lines: calculated transmission for $L=1075.6 \mathrm{~mm}, R_{1}=0.23, R_{\mathrm{Q}}=0.988$.

In Fig. 10, the measured transmission functions are plotted. A good agreement with the calculated data for a resonator length of $L=1075.6 \mathrm{~mm}$, a grating with $R_{1}=$ 0.23 , and a resonator round-trip efficiency of $R_{\mathrm{Q}}=0.988$ was obtained. Note that these results should not be used to infer the power transmission efficiency, as standing waves and drifts during the measurements do not allow sufficient amplitude accuracy. Calorimetric measurements to obtain precise data on power transmission are in preparation.

If the diplexer is used for in-line ECE experiments, then the power is directed via the resonant channel to the launcher, while the ECE signal is received via the nonresonant channel in backward direction, as sketched in Fig. 11. For this case, the isolation of the receiver port wrt. the gyrotron is an essential feature.

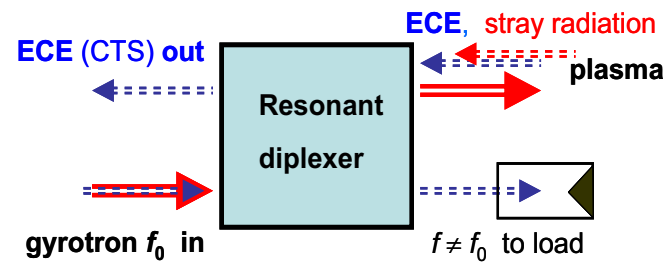

Figure 11. Sketch for in-line diagnostic experiments. The highpower ECRH is fed through the resonant channel, while the low-power diagnostic signal is received via the broadband nonresonant channel in backward direction.

The isolation was tested by measuring the power in the $\mathrm{HE}_{11}$ mode (i.e. with a matched receiver) in the isolated port for different loads at the outputs. Results similar to those for MC III [11] were obtained, as seen in Fig. 12. For matched (i.e. non-reflecting) outputs, a very high isolation of $>60 \mathrm{~dB}$ (noise level) is obtained. If a $100 \%$ reflection from a plane mirror, i.e. in $\mathrm{HE}_{11}$ mode, occurs, the power received in the isolated port is given by the product of the transmission functions, i.e. in the worst case is only $6 \mathrm{~dB}$ below the input power. In reality this improbable case occurs only when a plane vacuum shutter in front of launcher is closed. 


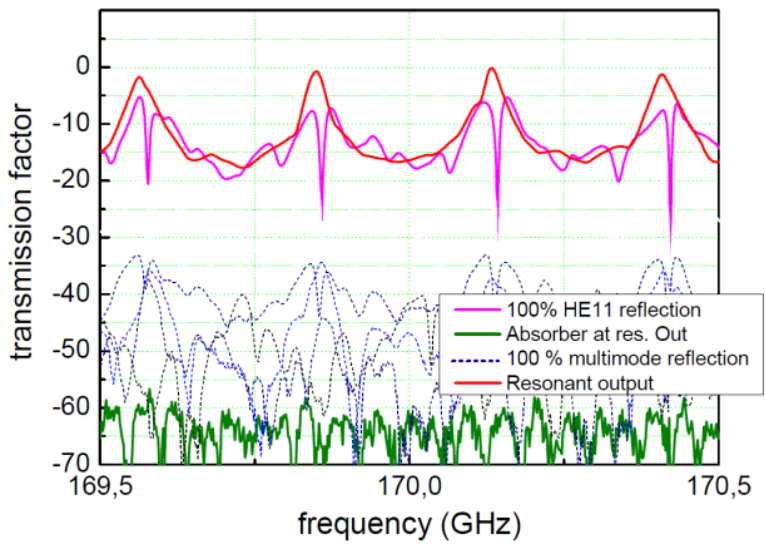

Figure 12. Test of the isolation of the backward non-resonant backward channel wrt. the input for an absorbing surface of $1700 \mathrm{~cm}^{2}$ ( $c f$. Figure 9). Cases shown are for (i) $100 \% \mathrm{HE}_{11}$ reflection (magenta line), (ii) $100 \%$ multimode reflection (dotted lines), and (iii) no reflection from the launcher (green solid line, for perfect absorption in the plasma). For reference, the transmission of the resonant output (red) is shown.

In the more practical case, where a reflection (e.g. from the plasma vessel or an arc) occurs in multi-mode (simulated by a $100 \%$ power reflection from a crumpled aluminium foil), the isolation is $\geq 40 \mathrm{~dB}$. The resulting power level seems sufficiently low for conventional notch filters, which have to be used additionally in front of sensitive receivers.

An essential feature of ring resonators is the suppression of wrong modes in the transmission system. Spatial mode filtering in the non-resonant channel due to truncation of the transmitted multi-mode beam at the mirrors in the diplexer will damp especially the veryhigh-order modes (VHOMs). From the design (ratio of absorber area to waveguide aperture), the suppression of modes with Brillouin angle $\theta>23^{\circ}$ is estimated to be $15 \mathrm{~dB}$.

The mode purification in the resonant channel is due to spatial mode filtering of VHOMs similar to the nonresonant channel. In addition, all wrong modes are attenuated owing to the different resonance frequencies of higher-order modes. The expected average suppression $S$ is given basically by the ratio of the width of the resonance to the free spectral range $\mathrm{c} / L$, and can be approximated as $S=0.6 \cdot R_{1}+0.3 \cdot R_{1}{ }^{2}$. For the present design with $R_{1}=0.23$, this yields $8 \mathrm{~dB}$ of average wrongmode suppression. Experiments to demonstrate this feature are going on.

\section{Summary}

The development of resonant diplexers for ECRH has advanced further. High-power applications have been demonstrated, and the development of an evacuated diplexer for $170 \mathrm{GHz}$ is ongoing. First low-power tests show high performance in agreement with theory; further tests on power transmission efficiency are in preparation.
High-power tests are envisaged at the gyrotron and transmission test stand at JAEA, Naka.

The application of the diplexer MkIIa for plasma experiments showed encouraging results concerning inline ECE [12] and NTM stabilization. However, these experiments show that further optimization of the resonator control system is needed. Concepts for robust controls have been tested successfully. Therefore, new experiments with improved resonator control and better auxiliary components are planned for ASDEX Upgrade.

\section{References}

1. W. Kasparek et al., Fusion Eng. Design 84 (2009) 1002 1005

2. M. I. Petelin, AIP Conference Proceedings 691 (2003) 251-262

3. M. A. Henderson et al., Nucl. Fusion 48 (2008) 054013

4. M. Maraschek, et al., Phys. Rev. Lett. 98 (2007) 025005

5. W.A. Bongers et al., Fusion Sci. Technol. 55 (2009) 188203

6. W. Kasparek et al., Fusion Sci Technol. 59 (2011) 729 741

7. N. Doelman, et al., Proc. of the 17th Joint workshop on ECE and ECRH (EC-17), Deurne, 2012.

8. J. Stober et al, poster, this conference

9. J. Stober et al, talk, this conference

10. D. Wagner et al., Fusion Sci. Technol. 52 (2007) 313 - 320

11. W. Kasparek, et al., Proc. of 17th Workshop workshop on ECE and ECRH (EC-17), Deurne, 2012.

12. H. van den Brand et al., this conference. 